\title{
Stability in chemistry and biology: Life as a kinetic state of matter*
}

\author{
Addy Pross \\ Department of Chemistry, Ben-Gurion University of the Negev, Beer Sheva, 84105, \\ Israel
}

\begin{abstract}
Despite the considerable advances in our understanding of biological processes, the physicochemical relationship between living and nonliving systems remains uncertain and a continuing source of controversy. In this review, we describe a kinetic model based on the concept of dynamic kinetic stability that attempts to incorporate living systems within a conventional physicochemical framework. Its essence: all replicating systems, both animate and inanimate, represent elements of a replicator space. However, in contrast to the world of nonreplicating systems (all inanimate), where selection is fundamentally thermodynamic, selection within replicator space is effectively kinetic. As a consequence, the nature of stability within the two spaces is of a distinctly different kind, which, in turn, leads to different physicochemical patterns of aggregation. Our kinetic approach suggests: (a) that all living systems may be thought of as manifesting a kinetic state of matter (as apposed to the traditional thermodynamic states associated with inanimate systems), and (b) that key Darwinian concepts, such as fitness and natural selection, are particular expressions of more fundamental physicochemical concepts, such as kinetic stability and kinetic selection. The approach appears to provide an improved basis for understanding the physicochemical process of complexification by which life on earth emerged, as well as a means of relating life's defining characteristics-its extraordinary complexity, its far-from-equilibrium character, and its purposeful (teleonomic) nature- to the nature of that process of complexification.
\end{abstract}

Keywords: Emergence of life; kinetic stability; kinetic state of matter; replicative chemistry; replicator space; biological complexification; teleonomic character; natural selection.

\section{INTRODUCTION}

Matter manifests itself in a variety of forms, primarily as the three traditional thermodynamic states of matter-solid, liquid, and gas. For any substance, these three states, as we well know, differ dramatically in their physical characteristics. However, thanks to well-established chemical principles, the physicochemical relationship between the three states, the reasons for the profound differences in their physical properties, and the rules that govern the interconversion of one state to another, are well understood.

But then there are living systems. A living cell is in a sense just one further form of material organization. A prokaryotic cell may be thought of as no more than an aqueous solution of several thousand chemicals and a few organized molecular assemblies contained within a membrane structure that

*Paper based on a presentation at the $17^{\text {th }}$ International Conference on Physical Organic Chemistry (ICPOC-17), Shanghai, China, 15-20 August 2004. Other presentations are published in this issue, pp. 1807-1921. 
is itself just a further kind of molecular assembly. This form of material organization also displays certain unique characteristics which, as we are well aware, are strikingly different to the ones exhibited by the thermodynamic states of matter mentioned above. However, in sharp contrast to the well-understood relationships between the traditional states of matter, the relationship between living systems on the one hand and those traditional states of matter on the other, remains confused and a subject of endless controversy. Just what is it about living systems that makes them living? It is quite paradoxical that despite the spectacular advances in molecular biology that have taken place during the last half-century, a coherent physicochemical framework that comfortably accommodates both animate and inanimate matter remains elusive. Recent advances in complexity theory, while opening new avenues for exploring the nature of living systems (for recent reviews, see refs. [1,2] and refs. therein), do not seem, as yet, to have resolved the fundamental issues.

Solids, liquids, as well as living systems are molecular aggregates-that is clear. But in contrast to inanimate solids and liquids, living systems are distinguished by three particularly striking characteristics. First, living systems display an extraordinary complexity that manifests itself both in a static, structural sense as well as a dynamic, reactivity sense. Regular thermodynamically driven aggregation of the kind we see in traditional solid and liquid states does not lead to that degree, or that type, of complexity. What was the driving force for the emergence of this unique kind of complexity? Second, living systems are far-from-equilibrium systems that must constantly tap into some external source of energy in order to maintain that far-from-equilibrium state. Failure to obtain a continuing supply of energy necessarily leads the animate system toward equilibrium - to death. Inanimate systems on the other hand, though not necessarily in an equilibrium state, do at all times tend toward that lower Gibbs energy state. Clearly, the thermodynamic pattern of behavior expressed by animate as opposed to inanimate systems is quite different and raises the question as to how, from a thermodynamic point of view, the emergence of energy-consuming, far-from-equilibrium systems would arise in the first place.

Third, and most strikingly, there is the teleonomic character of living systems - that sense of purpose that all living systems exhibit. As Kauffman [1] has put it, any living system acts as an "autonomous" agent, a system that acts on its own behalf. But how is it at all possible for a chemical system to act on its own behalf? Or, as Monod [3] put it several decades ago: How could projective systems have emerged from an objective universe? (projective systems being defined as those involved in furthering some project - to hunt for food, to seek a mate, etc.). Monod went so far as to term this dilemma the "central problem of biology".

Not surprisingly, the above questions have been the focus of considerable interest over past decades and have led to several distinct schools of thought. The "replication first" school of thought, pioneered by Eigen [4], proposes that life began with a replicating molecule, possibly RNA-like, which then underwent some process of complexification. This approach is questioned by some [5] because it has been argued that the likelihood for the emergence of such a molecule is remote, and, in any event, it is unclear how such a molecule would have managed to become transformed into the highly complex dual world of nucleic acids and proteins. An alternative school of thought, based on the relatively new area of complexity theory [1,2], argues that life is an emergent property of complex systems and that uncovering the rules that govern those kinds of systems will throw additional light on the life issue. But, here too there are some fundamental problems. Given that it is still unclear how the rules of complexity operate, not just in living systems, but in complex systems generally [6], the applicability of complexity theory to living systems has yet to be substantiated.

It is the purpose of this review to explore some of these issues by building on Eigen's "replication first" model and the kinetic approach to living systems that we have developed recently [7-10]. Given our attempt to bridge between chemistry and biology, a key strategy will be to consider both chemical and biological systems within the same terms of reference, even though the two sciences generally operate on the basis of different methodologies and terminologies. We begin, therefore, by considering the concept of stability within chemistry and biology, and the difference in the term as it applies in the two areas. As we shall see, all chemical systems, both animate and inanimate, tend to 
undergo change in a manner that enhances their stability, but it is the nature of the stability that characterizes chemical systems within these two worlds, that is quite distinct. In a nut-shell, our thesis states that the stability that governs the reactivity patterns of replicative systems-both chemical and biological-is of a kinetic kind, and stems from the unique kinetic character of autocatalytic processes. By contrast, the stability that governs the reactivity patterns of nonreplicating chemical systems-so-called "regular" chemical systems-is thermodynamic in nature. We believe it is the distinction between these two kinds of stabilities that leads to the dramatically different reactivity patterns and material characteristics that we observe in the worlds of animate and inanimate systems.

\section{DISCUSSION}

Any attempt to relate animate and inanimate must build on certain base assumptions, so let us begin by specifying the key ones that we have utilized. First, we assume animate and inanimate matter are directly related in some manner, based on the conviction that animate matter emerged from inanimate matter, and that this transformation took place by some physicochemical process of complexification on the primordial earth after its formation about 4.5 billion years ago. The possibility of panspermia is generally discounted, and in any case, a belief in panspermia merely changes the location of the initial complexification process, so the need to explain the process of emergence, whether on earth or elsewhere, remains.

Our second base assumption is to presume that the existing laws of physics and chemistry can explain the process of complexification that inanimate matter must have undergone in order to have generated chemical systems with the unusual characteristics mentioned above. After all, living beings are nothing more than physicochemical systems, so it seems reasonable to believe that the existing laws of physics and chemistry, which account satisfactorily for all other known physicochemical processes, should be able to account for the emergence of living systems as well. An alternative view, that as yet undiscovered scientific principles may lie behind the life phenomenon [1], seems to us unjustified given our current mechanistic understanding of biological systems and the broad recognition that the chemical processes associated with living systems are ultimately nothing more than networks (albeit highly complex ones) of "regular" chemical reactions.

Third, while the transition from inanimate to animate must have followed some particular mechanistic pathway, one we might term historic, we presume that the principles that governed that particular process were ahistoric. By this, we mean that the precise chemical composition of the primordial system and the mechanistic path that it happened to have followed were circumstantial, that the physicochemical principles that could explain the process of biological complexification should not be associated with just that single chemical system following that particular mechanistic path. We presume that under appropriate reaction conditions other chemical systems could, at least in principle, also undergo the special kind of complexification we associate with living systems. The basis for this third assumption rests on our general experience with chemical systems, where we find that their structural and reactivity properties are generally associated with material categories. Thus, for example, certain categories of materials tend to crystallize, some to conduct electricity, some have the propensity to polymerize, and so on. So, given the fact that at least one kind of chemical system (the nucleic acid-protein system) was able to complexify in the biological direction, we would presume that other chemical systems would also belong to the category of materials with the propensity to complexify in the biological-like direction. Addressing the emergence of life problem in this more general ahistoric manner has, in our view, two advantages. First, we would argue that a proper understanding of some particular historic process cannot be obtained without a proper appreciation of the underlying principles associated with that process [8]. Second, tackling the ahistoric question has the advantage of freeing us from the multitude of historic uncertainties associated with the actual process by which life on earth emerged. At least initially, we would not need to address the difficult historic questions, such as: Where on earth did life begin?, What were the reaction conditions on the prebiotic earth?, What was the struc- 
ture of the first replicating system?, etc., questions that are highly controversial in their own right. So let us now proceed by addressing a central theme of our argument—-the nature of stability.

\section{Nature's drive toward stable systems}

Dawkins [11] has alluded to a fundamental law of nature, one that applies to both the biological and the broader physicochemical world-survival of the most stable, i.e., the universe tends to be populated by stable things, where the term "stable" is used in the sense of persistent, unchanging with time. Dawkins goes on to point out that the Darwinian principle, survival of the fittest, then just becomes a special case of that broader law since fit individuals and fit species are more likely to survive, and therefore to persist.

Within the inanimate world, survival of the most stable as a general operational principle is certainly valid. In fact, the phrase expresses in a rather simple-minded way what is generally considered to be one of the most fundamental laws of physics and chemistry-the Second Law of Thermodynamics. The Second Law, which specifies the direction that all spontaneous irreversible processes must follow, states (in one of its several possible formulations) that any isolated physicochemical system is driven toward its lowest Gibbs energy (equilibrium) state. So, in attempting to relate animate and inanimate systems, we can initially point out a common feature: within both animate and inanimate worlds there is a drive toward greater stability though, as we will now discuss, the nature of the stability within an inanimate physicochemical context is quite distinct to the one that generally applies within the biological world.

A biological system, though stable in the sense that it maintains itself over time-in some case over billions of years-is actually unstable from a thermodynamic point of view in that it must tap into a constant source of energy, either chemical or photochemical, in order to maintain that far-from-equilibrium state essential to all biological function. Clearly, the stability that living systems exhibit is not thermodynamic in kind, and is only reflected in their persistent nature. Indeed, certain biological species, such as blue-green algae, would have to be characterized as extremely stable-colonies of such species have populated the earth with little change for some 3.5 billion years. Pandas, on the other hand, could be classified as unstable; without appropriate conservation measures, this species is likely to disappear before long. The point we are trying to make at this stage of the argument is just that there are different kinds of stability in nature, and each kind of stability, whether biological or chemical, requires definitive physicochemical characterization.

\section{Kinetic and thermodynamic stabilities}

In a general nonscientific context, we tend to associate stability with persistence, that is, an entity would be classified as stable if it is persistent (i.e., it does not change with time, though, of course, the length of time used to determine stability is necessarily a relative one). In a physicochemical context, however, stability is more explicitly defined and we traditionally distinguish between two kinds of stability-kinetic and thermodynamic. Consider, for example, the exergonic reaction of hydrogen and oxygen gases to yield water. Since that reaction is spontaneous but the reverse reaction is not, we state that $\mathrm{H}_{2} \mathrm{O}$ is thermodynamically stable while the $\mathrm{H}_{2}-\mathrm{O}_{2}$ gas mixture is thermodynamically unstable. However, the system comprising a $\mathrm{H}_{2}-\mathrm{O}_{2}$ mixture can be extremely stable in the sense that it can be extremely persistent, and indeed, under appropriate conditions such a mixture can be maintained almost indefinitely. In order for reaction to take place, some form of activation, provided by a spark or appropriate catalyst, is necessary. Thus, we term a $\mathrm{H}_{2}-\mathrm{O}_{2}$ mixture (under appropriate conditions) as kinetically stable because it is the high kinetic barrier to reaction that prevents the chemical reaction from taking place. Note that kinetic stability, in contrast to thermodynamic stability, is circumstantial as it is a property of the system and its immediate environment. Kinetic stability depends not just on the system itself, but on 
factors extraneous to the system. For this reason, kinetic stability cannot be classified as a state function (in contrast to thermodynamic stability).

\section{Dynamic kinetic stability}

The above description of kinetic and thermodynamic stability in chemistry is well established, but is inadequate for describing the stability (persistence) associated with replicating systems. The process of replication is unique and leads to stability of a different kind, one that derives from the kinetic consequences of replication. Let us clarify this point.

A single molecule replicating just 79 times generates a mole of material $\left(2^{79} \sim 6.10^{23}\right)$ while a further 83 replications would, at least in principle, lead to a mass the size of the earth. Clearly unchecked replication, like any other autocatalytic process, is unsustainable. Thus, any realistic kinetic description must recognize the limitation of available resources and the balance that must be established between formative and decay processes. Accordingly, one widely used kinetic formulation going back to the early $20^{\text {th }}$ century $[12,13]$ is given by

$$
\mathrm{d} X / \mathrm{d} t=k M X-g X
$$

where $X$ is the replicator concentration, $M$ is the concentration of building blocks from which the replicating system is composed, and $k$ and $g$ are the rate constants for replicator formation and decay, respectively. The key feature of this equation (and others of its kind) is that the replicator is undergoing competing processes of formation (the $k M X$ term) and decay (the $g X$ term), with a steady state being achieved if and when those two rates are equal (i.e., when $\mathrm{d} X / \mathrm{d} t=0$ ). Thus, replicators capable of maintaining a significant steady-state population could, by definition, be classified as kinetically stable in that a persistent population of replicators is present, even though the individual identities of that system are undergoing constant change. We see, therefore, that kinetic stability can be of two distinct kinds. It can be of the static kind, as reflected in a hydrogen-oxygen gas mixture, but it may also be of a $d y$ namic kind, as reflected in a replicating system.

Our description of what we might term dynamic kinetic stability [9] leads to a striking consequence. When we say a chemical system is stable, this normally means that the system remains unchanged because it fails to react, whether for kinetic or thermodynamic reasons. But an entity that has the special characteristic of being able to make copies of itself may be stable for a quite different reason. A replicating system may be stable, not because it does not react, but rather because it does! Its reaction-to make copies of itself, and at a rate that may be exponential. Thus, there is a unique kind of stability - a dynamic stability, associated with things that can make more of themselves!

Having extended the term kinetic stability to replicating systems, it would be useful to be able to quantify the concept in some way. A simple measure of the dynamic kinetic stability of a replicating system would be the size of the equilibrium population of replicators that has been established and can be maintained. The larger that population, the greater the ability of that replicating system to survive any perturbation on the system, and hence, the greater its kinetic stability. Thus, a replicating system that can only maintain a small equilibrium population would be classified as being of low kinetic stability, while one that cannot maintain a steady-state population at all (due to its tendency to degrade more rapidly than it can replace itself through replication), would be classified as kinetically unstable. In such cases, the population numbers may well drop to zero, i.e., such species are likely to become extinct [9].

\section{Transitions in replicator space}

Of course, the fact that some replicating molecule can react to make copies of itself and may, thereby, display stability of a dynamic kinetic kind, does not make that molecule alive, nor explain how or why that molecule became transformed into a simple living cell. But as we will now argue, the fact that the stability of a replicating system is of a dynamic kinetic kind can have profound chemical consequences. 
Chemistry deals with the structure of matter and the rules that govern material transformations. For "regular" chemical systems, the selection rule that governs the transformation of one chemical system into another is well known-it is a thermodynamic one, as dictated by the Second Law. Thermodynamically less stable systems tend to become transformed into thermodynamically more stable ones. Of course, kinetic factors influence the rate at which such transformations take place, but those kinetic factors are secondary to the thermodynamic ones; if the transformation is thermodynamically unfavorable, then no combination of kinetic factors can override the thermodynamic directive.

We have recently suggested [9] that the selection rule for the interconversion of replicating systems is qualitatively different to the one that governs the reactions of "regular" nonreplicating systems, in that it is predominantly kinetic rather than thermodynamic (though the Second Law is, of course, fully obeyed). The basic idea is readily demonstrated. Consider the situation in which an existing replicator $X_{1}$ undergoes a process of imperfect replication leading to the generation of a second replicator $X_{2}$. What will be the chemical outcome for two competing replicators, each obeying the kinetic scheme of eq. 1 (depicted in eqs. 2 and 3)?

$$
\begin{aligned}
& \mathrm{d} X_{1} / \mathrm{d} t=k_{1} M X_{1}-g_{1} X_{1} \\
& \mathrm{~d} X_{2} / \mathrm{d} t=k_{2} M X_{2}-g_{2} X_{2}
\end{aligned}
$$

As Lifson pointed out several years ago [14], the steady-state solution that results is one in which one of the replicators, say $X_{1}$, will tend toward some limiting concentration, while the concentration of the second replicator will drop to zero. In other words, two competing replicators obeying the kinetic scheme of eq. 1 cannot coexist; one of the replicators - the one we would define as kinetically more stable-drives the second replicator-the kinetically less stable one, into extinction. Note that this result in no way depends on the relative thermodynamic stabilities of the two replicators. The result is a kinetic one and derives from the kinetic conditions that have been imposed on the system.

This kinetic result, if it were to apply to replicating systems generally, would have profound consequences. Consider all replicating entities as comprising elements of a replicator space [9]. Any mutation in one replicator leading to the formation of some other replicator would be considered a transition in replicator space. If we consider eq. 1 as a general kinetic model for replicator formation and decay, then the only successful transitions in replicator space would be those that lead to replicators of higher kinetic stability. If a kinetically less stable replicator were somehow formed, then, given the kinetic result we have described earlier, it would simply disappear with time. Thus, the transition between two connected elements in replicator space would effectively only take place in one direction-the direction leading to replicators of greater kinetic stability.

Of course, the all-or-nothing kinetic result described above derives from the simple kinetic formulation of eq. 1, and other kinetic schemes would necessarily lead to other kinetic patterns [15]. Coexistence, for example, is the observed kinetic outcome when a more complex kinetic scheme that incorporates rate-limiting duplex dissociation is utilized [16]. Indeed, the very diversity of the biosphere might suggest that coexistence, rather than an all-or-nothing outcome, is the correct kinetic outcome for competing replicators. But that biodiversity is somewhat misleading. It is a widely accepted principle of ecology that two similar species, following similar life styles, and competing for the same limited resources, will not coexist - the more successful species will tend to drive the less successful one into extinction [17]. And indeed, returning to molecular replicators, more elaborate kinetic modeling of competing RNA oligomers has recently concluded [18] that a subset of polyribonucleotide chains is selected for, while less efficient ones become extinct, reaffirming in broad terms the simple kinetic prediction of eq 1 . Thus, despite the possibility of significant variability in the kinetic schemes that competing replicating systems may exhibit, it seems that the dynamic equilibrium that is established between competing replicators, whether molecular or complex, is inherently fragile. The process of mutation necessarily leads to a diversification of replicators, both in their structure and in their (kinetic) stability, and once a number of replicating systems emerge, all competing (directly or indirectly) for the 
same limited resources, there will be a general tendency for kinetically more stable replicators to displace kinetically less stable ones, and this is true at both the biological and the chemical levels. Thus, the biological term less fit to more fit, when translated into physicochemical terminology applicable to both chemical and biological entities, becomes kinetically less stable to kinetically more stable [9].

\section{Nature of biological complexification}

On the basis of the replicator space selection rule that we have just described, let us now attempt to better understand the nature of biological-like complexification, as opposed to traditional thermodynamically driven complexification. If, as we believe, life emerged from an inanimate environment, then it follows directly that inanimate matter under appropriate conditions can be driven to complexify in the biological direction.

We start with the observation that complexification as a process lies at the heart of evolutionary theory. It is widely accepted that eukaryotic life forms evolved from simpler prokaryotic life and multicell life forms from single-cell ones, i.e., early life was relatively simple and with time became more complex. However, in these particular instances, we are referring to biological (evolutionary) complexification as opposed to chemical (emergent) complexification (the process in which inanimate matter began to complexify in the biological direction). The question then arises as to whether the principles responsible for biological complexification on the one hand, and for chemical complexification on the other, are not in some way related. Eigen believed they were and that idea is, in fact, the essence of Eigen's thesis on the origin of life-that the Darwinian process of natural selection and survival of the fittest began to operate on some inanimate replicating system, and once some early life form emerged, continued to operate at the biological level [4]. Certain aspects of the classic RNA oligonucleotide replication experiments carried out by Spiegelman [19] did, in fact, support Eigen's hypothesis. Those experiments revealed that longer, slower segments evolved into faster, shorter ones, resulting in an evolutionary process in which a 4000-unit RNA oligonucleotide eventually shortened to one just several hundred units long. But this important result, though demonstrating unequivocally that molecular replicators follow a kind of Darwinian selection, did not show a process of complexification, or indicate why such a process might take place. The physicochemical principles that would have governed that initial complexification process-one which led a molecular replicator (mass ca. $10^{-21} \mathrm{~g}$ ) to become transformed into a replicating assembly (say, a prokaryotic cell of mass ca. $10^{-9} \mathrm{~g}$ ), remain unclear. Natural selection and survival of the fittest are biological terms, so the way they would induce a process of complexification at the molecular level is not obvious.

We recently suggested that the different selection rule in replicator space compared to "regular" chemical space may help explain the nature of the complexification process of replicating systems, as well as the possible reasons for that process being so different to "regular" thermodynamic aggregation (crystallization, vesicle and micelle formation, etc.) [10]. Building on the Eigen assumption that life began with the emergence of some replicating molecule, the ahistoric question that needs to be addressed is: Why would a one-molecule replicator tend to complexify, say, into a two-molecule replicating assembly? Is there some fundamental driving force of a nonthermodynamic nature that might lead to biological-type aggregation? We believe the answer is yes and point to the kinetic stabilities of various replicating systems to support that view. Consider first the high kinetic stability of phages and viruses.

A phage (or virus) may be simplistically viewed as a two-molecule replicating system-an aggregate comprising a nucleic acid and a protein molecule, though in practice more than one protein structure and more than one molecule of each structure may be involved. Of course, in an appropriate biotic environment phages are potent replicators. The high replicating capability of the aggregate comes about through a symbiotic interaction between its two components: the protein component protects the nucleic acid component from chemical attack and facilitates its entry into the host cell. The cell's replicating machinery is then taken over, leading to the synthesis of the phage components which then self- 
assemble, leading to the formation of a large number of new phage entities. The point is that the two phage components, when separated, are kinetically unstable entities, since, as separate entities each is unable to replicate under the same conditions. It is only the viral aggregate in toto that is capable of effective replication, i.e., exhibits high kinetic stability. Thus, even though the actual force that leads to aggregation is a thermodynamic one, the fact that the resulting aggregate is replicative means the effective driving force is kinetic - the kinetic power of autocatalysis [7].

Of course, the example of phage replicative capability is only applicable in a biotic environment where the phage can exploit the metabolic and replicative machineries of its host cell. We have provided an example from a biotic environment because that is the environment we are familiar with! Clear examples from prebiotic times would be somewhat harder to produce! We would argue however that the symbiotic principle that forms the basis of the example would apply in any environment-biotic or abiotic. For replicating systems, any process of self-assembly that leads to the formation of kinetically stable aggregates would be kinetically selected. That process would be part of the multidimensional exploration of replicator space whereby all possible degrees of freedom-material, spatial, even temporal-are exploited in the continual search for kinetically stable replicators.

There is indeed broad empirical evidence that supports the idea that the process of molecular complexification leads to the formation of replicators of enhanced kinetic stability. The pioneering work of Orgel [21] and von Kiedrowski [22] demonstrated that single-molecule replication is not a facile process and attempts to carry out such reactions without enzymatic catalysis have met with only limited success-molecular replicators tend to be kinetically unstable. By comparison, complex replicators (e.g., bacteria) display remarkably high kinetic stability and replicate prodigiously under highly variable conditions, including those considered extremely hostile, with no need for a sophisticated laboratory or human direction. Thus, the fact that complex replicators are generally kinetically more stable than simple ones, would mean that successful transitions in replicator space would tend to be those that lead to an increase in complexity, that is, the (effective) kinetic driving force tends to transform less stable replicators that are simpler, into more stable ones that are complex. \{Note, however, that not all transformations in replicator space necessarily involve complexification. If within the general exploration of replicator space a specific process of simplification occurs that results in an increase in kinetic stability, then clearly that process will also be kinetically selected. Our point is that greater kinetic stability is associated with greater complexity in the same way that the process of climbing a mountain generally involves following ascending paths, though occasionally short, descending ones may be involved (see also ref. [20])\}.

The above discussion may provide additional insight into the fundamental question initially posed by Dyson [23] and repeated by Kauffman: Why is it that even minimal life is so complex? We would argue that just as "regular" chemical space tends to be occupied by thermodynamically stable entities, so replicator space would tend to be populated by kinetically stable entities. Less complex precursor replicators, that would have preceded the more complex established replicators, being less stable, would have been less likely to survive. Just as thermodynamically stable entities are frequently formed through the transient formation of thermodynamically unstable intermediates of which no trace remains, so the formation of kinetically stable entities from kinetically unstable ones could also be expected to occur without remnants of those unstable precursors being observed. We also need to bear in mind that in prebiotic times a less complex replicating entity, sufficiently stable to survive in that early environment, might well be less capable of surviving in the current highly diverse and richly populated biosphere. Recall that kinetic stability is not a state function and depends crucially on factors extraneous to the system itself, so a replicating system that could have been stable in an early biotic period may well be unstable in today's environment. The situation is analogous to planting a new plant in an established garden vs. a plant-free area-it is more difficult for plants to successfully take root in established areas compared to plant-free areas. We believe that such an explanation for the observation of the high minimal level of complexity in extant life to be more reasonable than the proposal that the transition from non-life to the enormous complexity of even the simplest life forms was accomplished in a single "crys- 
tallization of life" leap [1]. To the best of our understanding, there is no empirical or credible theoretical basis that supports the postulation of such an extraordinary event. To summarize, in today's highly diverse biotic environment we suggest that a relatively high level of complexity is required in order to reach the threshold level of minimal kinetic stability - the level that replicating systems need to function successfully (we exclude parasitic assemblies, such as viruses and phages, where a much lower level of complexity is required as they exploit elements of the host system's complexity). However, the absence of less complex entities in today's biosphere in no way implies that such entities were not formed transiently at an earlier phase of life's emergence.

The above considerations concerning biological complexification allow us to propose a general complexifying principle for molecular replicators expressed in physicochemical (rather than biological) terminology: If a molecular replicator X (schematically depicted in eq. 4), acts as a catalyst for the formation of some molecule (or molecules) $Y$ (schematically depicted in eq. 5) that self-assemble(s) with the replicator (eq. 6), such that the molecular assembly X//Y itself is both replicative and kinetically more stable than the replicating molecule $X$ alone, then sequences that facilitate the catalytic formation of such molecules will be kinetically selected. Thus, for example, $\mathrm{X}$ might be an oligonucleotide; $\mathrm{Y}$, an oligopeptide; A, B, C, activated nucleotides; L, M, N amino acids; and Z, a leaving group, such as pyrophosphate.

$$
\begin{aligned}
& \mathrm{A}+\mathrm{B}+\mathrm{C} \stackrel{\mathrm{X}}{\longrightarrow} \mathrm{X}+\mathrm{Z} \\
& \mathrm{L}+\mathrm{M}+\mathrm{N} \stackrel{\mathrm{X}}{\longrightarrow} \mathrm{Y}+\mathrm{Z} \\
& \mathrm{X}+\mathrm{Y} \longrightarrow \mathrm{X} / \mathrm{Y}
\end{aligned}
$$

Therefore, given the above set of reactions, the optimal replicator sequence is not necessarily the one that is the inherently the fastest, as might be inferred from Spiegelman's classic experiments [19]. Rather, over the longer term the optimal kinetic result would be to locate sequences that cocatalyze the formation of other materials capable of catalyzing the replication reaction (e.g., proteins for enzyme catalysis), as well as sequences that would catalyze the formation of materials that would reduce the rate of replicator degradation (e.g., amphipathic lipid molecules for membrane formation). Thus, through the establishment of nucleic acid sequences that code directly or indirectly for those ancillary materials, the various components of the replicating assembly become replicatively coupled [24]. That coupling ensures that the resulting aggregate is replicative as a holistic entity. The catalytic formation of materials that can aggregate with the molecular replicators, leading to assemblies with increased replicative capability and/or reduced degradative tendencies, would all contribute to enhancing the steady-state replicator population and hence its kinetic stability.

In concluding this section, it is interesting to note that there is a well-documented biological example of what we would consider this fundamental kinetic complexification principle. One of the key steps in biological evolution was the endosymbiotic transformation of prokaryotic life forms into eukaryotic life through the merging of two bacterial cells [25]. That merging process led to a more complex replicating assembly that turned out to exhibit high kinetic stability. Thus, the endosymbiotic process of biological aggregation is the biological analogue of the more fundamental process of molecular aggregation that we hypothesize would have taken place on the long road from replicating molecule to complex replicating assemblies. Both the process of emergence (i.e., chemical complexification) and that of biological evolution (i.e., biological complexification) manifest the same fundamental kinetic complexification principle - the exploration of replicator space for kinetically stable replicating assemblies. We believe a major experimental challenge is to demonstrate the operation of that complexification principle at the molecular level.

To sum up, we are proposing that it is the drive toward enhanced dynamic kinetic stability (by kinetic selection) that has induced the process of complexification, both at the chemical and the biological levels. The replicative reaction is a unique one in chemistry in that it is effectively driven by the 
enormous kinetic power of autocatalysis [7]. Accordingly, biological-like (kinetic) complexification is a feasible physicochemical outcome that can result from an ongoing process of (mutagenic) replication. Mutagenesis inevitably leads to a continual process of kinetic selection, initially within sequence space, subsequently within a more general replicator space, but at all times tending toward replicating systems of enhanced kinetic stability.

\section{Living systems as a kinetic state of matter}

The above discussion now enables us to provide a broad physicochemical distinction between living aggregates, such as bacterial cells, and inanimate aggregates such as crystals, vesicles, etc., and a basis for understanding their strikingly different characteristics. We would suggest that all living systems, from simplest prokaryote through to complex multicellular plants and animals, but also including parasitic species such as viruses and phages, may be thought of as manifesting a kinetic state of matter, as opposed to the traditional thermodynamic states of matter with which we are familiar [9,10]. (Before continuing, we should point out that the kinetic state is strictly speaking not a true state at all, but a pseudostate. A true state of matter is defined as one at equilibrium and characterized by certain state functions, while a kinetic steady state is not at equilibrium in a thermodynamic sense). So the term kinetic state of matter highlights the fact that replicating systems, animate or inanimate, by the very fact of being replicative, have the potential to access nonthermodynamic, kinetic steady states, resulting in the formation of aggregates that are stable, though the stability is kinetic in nature. Thus, there exists in nature a stability that is quite different to the conventional chemical stability we normally associate with an inherent lack of reactivity. For replicating systems, the stability is of a dynamic kinetic kind, one that derives from the special kinetic character of the replicative process. It is the immense kinetic power of autocatalysis inherent in all replicating processes that is the ultimate driving force for the generation of the complex physicochemical pattern that we label as life.

In concluding this section, we should point out that one of the most remarkable features of kinetic states of matter is that their dynamic kinetic character can manifest at several hierarchical levels, depending on the system's complexity. For molecular replicators, the dynamic character of the system is of course evident at just the molecular level. A steady-state population of molecular replicators is maintained through a continual process of molecular generation and degradation, one might say a molecular fountain in action! For the more complex cellular replicators, however, the dynamic character is evident at two levels-molecular and cellular. At the molecular level, key biomolecules are being continually turned over. For example, most cellular proteins have half-lives measured in hours, some as short as 11 minutes, due to a highly specific process of protein degradation and resynthesis [26]. This means that cellular protein, a primary constituent of all living cells, is effectively all replaced within several days - a further illustration of the molecular fountain in operation! But, of course, at the cellular level, constant turnover of entire cells also takes place-new cells are continually formed by cell division, while existing cells are continually degraded. And finally, when we turn to the even more complex multicell replicators - plants and animals-we now see continual turnover at three hierarchical levels - at the molecular level, the cellular level, and the organismic level. In the world of replicative kinetic states, continual turnover-at all hierarchical levels-is the name of the game!

\section{Physicochemical characteristics of kinetic states of matter}

Based on the above kinetic state description of living systems, we believe the most striking characteristics of living systems - their high complexity, far-from-equilibrium character, and teleonomic nature-can now be better understood. 


\section{High complexity and far-from-equilibrium character}

In stark contrast to typical inanimate aggregates, living systems are thermodynamically unstable, highly complex, far-from-equilibrium systems. As discussed above, the selection rule within replicator space is a kinetic one, in which kinetically less stable replicators tend to become transformed into kinetically more stable ones. Accordingly, replicator thermodynamic stability is not a necessary characteristic, nor even a desirable one. In fact the reverse appears to be true. It turns out that successful replicating systems (i.e., those exhibiting high kinetic stability) are highly complex and maintain a far-from-equilibrium state because these two characteristics both serve to enhance kinetic stability. Of course, in order to satisfy the thermodynamic requirements associated with maintaining far-from-equilibrium replicating systems, an energy-gathering capability must also be incorporated into the replicating assembly. That metabolic machinery is just an additional form of complexity that has been acquired by kinetic selection, one that enables other kinds of complexity (structural and informational) to increase despite the associated thermodynamic costs [8]. In replicator space, it is only kinetic stability that counts, provided, of course, that the thermodynamic ledger is properly balanced [9]. A general description of the kinetic process by which a nonequilibrium replicator might have been transformed into a far-from-equilibrium one has been discussed in an earlier paper [10].

\section{Teleonomic character}

Let us now turn briefly to what is possibly life's most striking characteristic-its teleonomic character (i.e., the ability of living systems to exhibit purposeful behavior) [27]. Regrettably, the very mention of the purpose word in a scientific context often raises cries of dissent as the term itself is commonly used in a nonscientific context, and what is worse, sometimes used in a scientific context, but in a nonscientific way. So let us first point out that eliminating the purpose word from biological thinking eliminates what is probably the most powerful organizing principle of biology, in particular, functional biology [28]. In recognition of that fact, it seems fair to say that modern biology is generally comfortable with the concept of purpose. As Pittendrigh put it several decades ago, biologists are now happy to say that "a turtle came ashore to lay her eggs", rather than "a turtle came ashore and laid her eggs" [27]. Nevertheless, the question how a chemical system can exhibit such character remains unresolved. While the general scientific community no longer believes that vital forces are needed to explain the phenomenon of teleonomy, the source of that character remains elusive.

One possible explanation for life's teleonomic character is that it is an emergent property associated with the highly complex nature of living systems. The very concept of emergence stresses that at different hierarchical levels of complexity, new system properties may manifest. While the complexity explanation does superficially appear to provide a scientific answer to the problem of teleonomy, there is an innate difficulty with this kind of explanation. Brushing the problem of teleonomy under the complexity carpet in some respects only serves to replace the now discredited and unscientific vital force by a concept that has yet to be unambiguously defined and whose governing rules remain to be established. We believe the kinetic approach can offer an alternative explanation for this most remarkable of life's characteristics.

Since teleonomic behavior remains a linchpin of all biological phenomena, we have recently proposed a model that attempts to relate that remarkable pattern of behavior to the kinetic nature of the replication process [29]. Our analysis sought to provide an answer to the question: How is it at all possible for a chemical system to act on its own behalf? Is there some physicochemical explanation for the observation that during the process of emergence, a so-called objective system [3] became transformed into a purposeful (teleonomic) one? The analysis began with a physicochemical characterization of teleonomic as opposed to nonteleonomic events, where the archetypal teleonomic event is taken to be cell replication. The key conclusion was that regular, nonteleonomic events are those whose primary directive is thermodynamic, while teleonomic events, at least at the cellular level, are those whose primary directive is kinetic. Identification of the primary directive in cell replication as kinetic was reached by considering the thermodynamic behavior of a living cell. During the process of bacterial replication 
in a growth medium of glucose and essential salts, just enough glucose is oxidized to carbon dioxide and water to enable the endergonic biosynthetic kinetic pathways leading to cell division to take place $[3,7]$. Thus, the biological system has evolved in such a way that the thermodynamic directive has taken on a role that is secondary and supportive to the primary kinetic one of cell replication [29]. In other words, during the process of life's emergence from objective to teleonomic, the relative importance of kinetic and thermodynamic factors in governing chemical processes was somehow inverted.

How did that inversion take place? We believe the transformation occurred, as for all other transformations in replicator space, through a process of kinetic selection. Since teleonomic replicating systems tend to be kinetically more stable than nonteleonomic ones (e.g., bacteria are kinetically more stable than molecular replicators), we believe the transformation from nonteleonomic to teleonomic was one with a clear physicochemical driving force. Specifically, the incorporation of an energy-gathering metabolic system into an evolving replicating system (by kinetic selection) $[8,29]$ would have transformed a nonmetabolic replicator, whose reaction would have been thermodynamically directed, into a metabolic replicator whose reaction would have become kinetically directed. Now, given our physicochemical definition of a teleonomic event described above, that incorporation would mean that an $o b$ jective replicator would have become transformed into a teleonomic one! In sum, the emergence of what we term purposeful behavior was just one further means by which greater kinetic stability for replicating systems was attained. A detailed analysis of this issue has recently appeared [29].

\section{Generalizing Darwinian concepts}

There is no argument that the principle of natural selection as described in Darwin's classic On the Origin of Species [30] has become biology's most fundamental and influential concept. Therefore, given our attempt to place animate and inanimate systems within a single physicochemical framework, the question arises as to how our ideas on kinetic stability and kinetic states of matter relate to that traditional Darwinian view. Let us now briefly consider this issue.

We noted earlier that Eigen's thesis on the origin of life [4] postulated a process of natural selection at the molecular level, thereby outlining a plausible mechanism for the emergence of life. Importantly, by suggesting that Darwinian selection was first operational at the molecular level, Eigen laid the foundation for a conceptual bridge linking chemistry and biology. But the goal of accommodating biological and chemical thinking within a common conceptual framework may also be sought in an alternative fashion. Rather than apply what is essentially a biological concept to the chemical level (as Eigen did), we can reverse the approach and attempt to apply established chemical concepts to the biological level. After all, biological systems are just complex chemical systems, so being able to successfully apply chemical concepts to biological systems would provide scientific understanding at a more fundamental level. Accordingly, we ask the following question: Could the biological principle of natural selection be viewed as a particular application of some broader physicochemical principle? As the previous sections may have suggested, we believe the answer to be yes, that Darwinian theory derives from a more general chemical principle whose roots lie within traditional chemical kinetic theory. Thus, our "kinetic state of matter" model of living systems leads us to suggest that biological natural selection is a particular case of kinetic selection, that the concept of fitness is a particular case of kinetic stability and survival of the fittest is a particular case of kinetic selection of the kinetically more stable [10]. Similar reasoning suggests that one can reorder the Darwinian principle with its purely biological focus: that living things replicate (imperfectly) and therefore evolve, to obtain a more general physicochemical principle that encompasses both animate and inanimate systems: that certain (imperfectly) replicating things can evolve (complexify) and therefore become living (if they are not so already). In the traditional biological (Darwinian) view, replication is considered a characteristic of life, while a broader physicochemical view, which incorporates within it that biological view, reverses the causal relationship and suggests life to be a set of characteristics that emanate from certain highly complex chemical replicators. In fact, we would go as far as to say that life is a particular expression of an area 
of chemistry-replicative chemistry, which remains largely unexplored at the level of simple replicating assemblies.

\section{Convergence and divergence in chemical space}

In this last section, we briefly point out a significant topological distinction between "regular" and replicator spaces and discuss some interesting consequences that follow from that difference. Transitions within "regular" space, being thermodynamically directed, are convergent in character, while transitions in replicator space, being kinetically directed, are divergent in character. Let us expand on the idea.

Consider a number of isomeric hydrocarbon-oxygen mixtures, all elements within "regular" chemical space. Such mixtures might undergo reactions along a variety of different pathways and through a variety of different intermediates. For example, in any particular case, elemental carbon or carbon monoxide may or may not form, depending on the particular reaction conditions, but regardless of the different mechanistic routes that the various systems might follow, all paths are directed toward the same thermodynamic sink - carbon dioxide and water. More generally, each and every chemical system, whatever its structure, is thermodynamically directed toward its particular thermodynamic sink at the particular conditions specified - a sink it shares with an infinite number of possible isomeric systems. Thus, in a broad sense, all transformations in regular chemical space are convergent as all isomeric systems are directed toward their common thermodynamic sink. The convergent pattern in "regular" space is represented schematically in Fig. 1a.

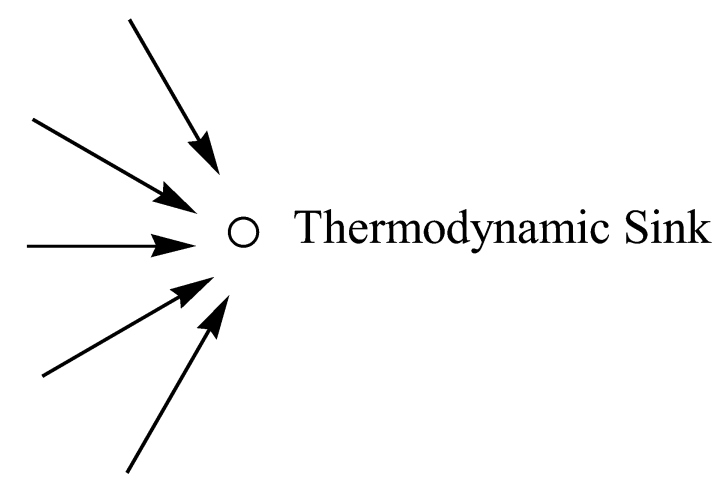

"Regular" (thermodynamic) Space

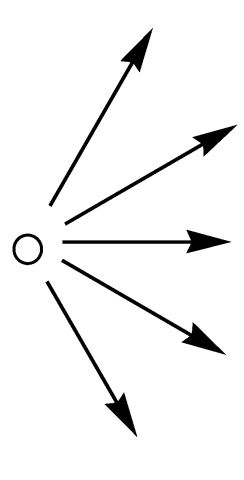

Replicator (kinetic) Space

\section{Convergent}

(a)

\section{Divergent}

(b)

Fig. 1 Schematic representation of branching patterns within (a) "regular" (thermodynamic) space (convergent), and within (b) replicator (kinetic) space (divergent).

By contrast, transformations in replicator space follow an opposite pattern, as illustrated in Fig. 1b. For transformations in replicator space, which as we discussed earlier lead toward systems of enhanced kinetic stability, there is no specific target of maximal kinetic stability because kinetic stability is not a state function. Kinetic stability depends on factors external to the system, and accordingly there is no single well-defined pathway to higher values. Thus, any given replicating system may potentially increase its kinetic stability in any number of different ways, so that each system within replicator space becomes a potential branching point for other kinetically stable systems. The result-within 
replicator space we observe a pattern of diverging pathways, as opposed to the pattern of converging pathways associated with transformations in "regular" chemical space.

Of course, the pattern of convergence in "regular" chemical space as well as the pattern of divergence in replicator space, manifest themselves through the progress of time. What this means, however, is that if we trace reaction pathways back in time, the patterns of convergence and divergence become reversed; a path that is convergent in the forward direction is necessarily divergent in the backward direction, and vice versa. Interestingly, it appears that this characteristic has practical consequences regarding our ability to make both predictive and historical statements regarding systems in the two spaces. Let us consider this idea in more detail, firstly, as it applies to replicator space.

We have already noted that replicator space going forward in time is divergent and that going back in time it is convergent. This convergence going back in time means it is easier to access historical information regarding precursor replicating systems because a converging path by definition is directed toward a limited number of primal points. In fact, given the fossil and genetic record, the current evidence supports the view that all living systems on earth descended from just one such primal system. In other words, inspection of the fossil and genetic record has enabled us to explore our evolutionary history - from early prokaryotic life through to recent multicell life forms—with considerable success, but this success has depended crucially on the convergent nature of replicator space as we go back in time. However, when we attempt to make predictive statements about replicator space the situation is reversed. The question as to where the future exploration of replicator space is likely to lead us is one which we cannot even begin to address; a diverging path, by definition, does not go anywhere in particular. The evolutionary future of replicating systems is effectively unknowable.

Applying the same thinking to the consideration of transformations in "regular" space leads to the opposite pattern. We can make reasonable predictive statements as to where a regular chemical system is directed (i.e., in a convergent direction toward its thermodynamic sink). However, making reliable statements regarding the identity of historical precursor systems in regular space is much more problematic. Going back in time toward possible precursor systems leads us in a divergent direction, to increasing numbers of potential precursor systems, thereby making the identification of an actual precursor system much more difficult. In sum, the different patterns of the two spaces-replicator and "regular"- -suggests that our ability to make either predictive statements regarding future transformations or historical statements regarding the nature of past transformations is greatly affected by the topology of the two spaces. A space that is convergent is inherently more informative in the convergent direction, whether in a predictive or in an historical sense, than a space that is divergent.

\section{CONCLUDING REMARKS}

In this review, we have postulated that the two physical manifestations of matter that we find around us - animate and inanimate-derive from the different kinds of stability that matter can express. Stability within the biological world derives from the nature of the replicating process: things that can make more of themselves will tend to abound, and, therefore, replicating systems reflect a stability that is kinetic in nature, a dynamic kinetic stability of the kind we find in a flowing river or a water fountain. By contrast, stability in the "regular" chemical world of nonreplicating entities derives from a lack of reactivity: things that do not react will persist. That lack of reactivity can derive from both thermodynamic and kinetic factors, but even when the stability is kinetic, it is of a static kind, and quite distinct from the dynamic kinetic stability associated with replicating systems. Cursory inspection of the world around us makes it quite evident that the stability associated with things that can make more of themselves is no less substantial than the stability of those things that do not react. Thus, in our attempt to place animate and inanimate within a common physicochemical framework, we are led to subdivide chemistry into two broad domains- "regular" (nonreplicative) chemistry and replicative chemistry. All replicative systems may usefully be thought of as occupying a structural space we term replicator space, while "regular" (nonreplicative) systems occupy a different space, one we might term "regular" chem- 
ical space. Observed physicochemical aggregation patterns within these two spaces are strikingly different because chemical transformations in the two spaces follow different selection rules. The selection rule in regular space is fundamentally thermodynamic, while that in replicator space is effectively kinetic, tending from kinetically less stable to kinetically more stable replicating entities. It is that kinetic selection rule that can explain the kinetically directed process of biological-type aggregation and complexification, as opposed to the well-understood process of thermodynamic aggregation that is characteristic of inanimate systems. Thus, our message is that all living systems are manifestations of an area of chemistry we would term replicative chemistry, and the unique characteristic of living systems can be understood in terms of their characterization as kinetic states of matter. Most importantly, however, it is through such a characterization of living systems that their main features become clearer. The extraordinary complexity of all living systems, their far-from-equilibrium state, and their teleonomic character all appear explicable when considered from this kinetic point of view [7-10,29].

Let us now briefly comment on how our perspective compares to the one that views living systems as an "emergent property" of complex systems. Proponents of this view, who base their ideas on the study of complex dynamical systems, such as Boolean networks, suggest that complex chaotic systems may spontaneously self-organize, so that life could have spontaneously "crystallized" out of an appropriately complex chemical mixture [1]. While that approach has been subject to broad criticism $[8,14,31,32]$, the study of complex systems as an exercise in applied mathematics has produced interesting insights that may have some bearing on the emergence of life problem. One profound conclusion to emerge from the study of complex systems is that simple rules can lead to complex behavior. That is readily discernible from the study [33] of cellular automatons, for example. So while it may well be true that complexity may come about for complex reasons, it is equally true that complexity may come about for simple reasons. Indeed, that is a key message of this review- that while life is undeniably complex, the principles that govern the emergence of living systems, together with their associated characteristics, are simple (or relatively so), and do not derive from complexity per se. It is our view that the key features that characterize living systems (at least at the single-cell level) are understandable in terms of the fundamental principles of chemistry that are used to explain all other chemical processes-a combination of kinetic and thermodynamic factors associated with mutagenic replicating systems-rather than deriving from the complex nature of the systems that happened to have emerged.

Of course, there remain many issues that require further clarification, even when historic uncertainties are put aside. We have attempted to describe the complexification principle by which a singlemolecule replicator would be driven to become, say, a two-molecule replicating assembly. However, there is as yet no direct empirical evidence to support that idea. It is only implied at the molecular level by the symbiotic interaction between viral components, and at the biological level by the endosymbiotic theory [25] that proposes that eukaryotic life came about through microbial association. A second point of uncertainty concerns the fact that complex replicating assemblies (cells) incorporate both static aggregates as well as dynamic reaction networks, so that leads us to the question as to which form of complexification would have emerged first. Could an emerging reaction network, such as hypercyclic organization of the kind originally hypothesized by Eigen and Schuster [34], lead to some form of physical aggregation and compartmentalization, or would the process have needed to begin with static aggregation, followed by the emergence of reaction networks within those static aggregates? The fact that small static replicating aggregates (viruses and phages) do exist, and the fact that it is difficult to envision precisely how the emergence of replicating reaction networks could have preceded the process of compartmentalization (a form of aggregation), seems to support the idea that static aggregation would have preceded the emergence of dynamic reaction networks. But these thoughts are speculative in nature and stem from the very limited amount of understanding we have regarding replicative chemistry at the molecular level. Indeed, it is paradoxical that while various aspects of replicative chemistry at the biological level are the focus of thousands of researchers in a variety of biological disciplines and lead to thousands of publications in dozens of journals, the study of replicative chemistry at the chemical level is still in its infancy. In particular, replicative chemistry beyond the single molecule, i.e., the study 
of simple replicative aggregates and minimal replicative networks, remains terra incognita though pioneering publications by Sievers and von Kiedrowski [35], Rebek et al. [36], Ghadiri et al. [37], and Chmielewski et al. [38], have begun to report on this virgin area of chemistry. The Holy Grail in this area, as outlined some years ago by Orgel [21], remains unchanged - the establishment of a general biology, a biology that is not necessarily linked to the specific biomolecules from which biological complexity is structured (nucleic acids, proteins, lipids, etc.), but one that will be based on the chemistry of the class of molecular replicators. It is, however, important to stress that for a biological-like system to emerge from any particular replicator, the property of self-replication would be a necessary, but not sufficient, condition. The class of molecular replicators capable of evolving into what we would consider to be living systems must also be able to induce, through their sequence-specific catalytic activity, the emergence of an entire repertoire of ancillary support materials kinetically selected for their ability to enhance the replicator kinetic stability. It would be the aggregation of those ancillary materials, all replicatively coupled to the molecular replicator, directly or indirectly, which would lead to the emergence of replicating assemblies and eventually to life.

\section{ACKNOWLEDGMENTS}

The author thanks Profs. Jan Engberts and Vladimir Khodorkovsky for most helpful comments.

\section{REFERENCES AND NOTES}

1. S. A. Kauffman. Investigations, Oxford University Press, Oxford (2000).

2. F. Capra. Emergence 4, 15-33 (2002).

3. J. Monod. Chance and Necessity, pp. 21-22, Random House, New York (1971).

4. (a) M. Eigen. Naturwissenschaften 58, 465-523 (1971); (b) M. Eigen. Steps Toward Life: A Perspective on Evolution, Oxford University Press, Oxford (1992).

5. R. Shapiro. IUBMB Life 49, 173-176 (2000).

6. S. Weinberg. NY Rev. Books 49 (16), (2002).

7. A. Pross. J. Theor. Biol. 220, 393-406 (2003).

8. A. Pross. Origins Life Evol. Bios. 34, 307-321 (2004).

9. A. Pross and V. Khodorkovsky. J. Phys. Org. Chem. 17, 312-316 (2004).

10. A. Pross. Origins Life Evol. Bios. 35, 151-166 (2005).

11. R. Dawkins. The Selfish Gene, p. 12, Oxford University Press, Oxford (1989).

12. A. J. Lotka. J. Phys. Chem. 14, 271 (1910).

13. A. R. Peacocke. An Introduction to the Physical Chemistry of Biological Organization, Chap. 4, Oxford University Press, Oxford (1989).

14. S. Lifson. J. Mol. Evol. 44, 1-8 (1997).

15. E. Szathmáry and J. Maynard Smith. J. Theor. Biol. 187, 555-571 (1997).

16. G. von Kiedrowski. Bioorg. Chem. Frontiers 3, 113-146 (1993).

17. R. Dawkins. The Blind Watchmaker, p. 239, Norton, New York (1986).

18. J. A. D. Wattis and P. V. Coveney. J. Phys. Chem. B 103, 4231-4250 (1999).

19. S. Spiegelman. American Scientist 55, 221-264 (1967).

20. The fact that viruses are kinetically more stable than their separate components does not necessarily mean that viruses emerged by a process that led from an individual molecular replicator to a viral assembly. In fact, the current view is that viruses resulted from postcellular evolution in what was most likely a process of simplification.

21. L. E. Orgel. Acc. Chem. Res. 28, 109-118 (1995).

22. G. von Kiedrowski. Angew. Chem., Int. Ed. Engl. 25, 932-934 (1986).

23. F. Dyson. Origins of Life, Cambridge University Press, Cambridge (1985).

24. V. Norris and I. Fishov. J. Biol. Phys. Chem. 1, 29-37 (2001). 
25. L. Margulis. Symbiosis in Cell Evolution, Yale University Press, New Haven (1981).

26. (a) A. Hershko and A. Ciechanover. Annu Rev Biochem. 67, 425-479 (1998); (b) J. M. Berg, J. L. Tymoczko, L. Stryer. Biochemistry, $5^{\text {th }}$ ed., p. 635, Freeman, New York (2002).

27. C. S. Pittendrigh. In Behavior and Evolution, A. Roe and G. G. Simpson (Eds.), pp. 390-416, Yale University Press, New Haven (1958).

28. E. Mayr. Toward a Philosophy of Biology, Harvard University Press, Cambridge (1988).

29. A. Pross. Origins Life Evol. Bios. 35, 383-394 (2005).

30. C. Darwin. On the Origin of Species by Means of Natural Selection, Murray, London (1859) [1964 facsimile ed.].

31. J. Maynard Smith and E. Szathmáry. The Major Transitions in Evolution, Freeman, Oxford (1995).

32. L. E. Orgel. Nature 358, 203-209 (1992).

33. S. Wolfram. A New Kind of Science, Wolfram Media (2002).

34. M. Eigen and P. Schuster. Naturwissenschaften. 64, 541-565 (1977).

35. D. Sievers and G. von Kiedrowski. Nature 369, 221-224 (1994).

36. (a) R. J. Pieters, I. Huc, J. Rebek, Jr. Angew. Chem., Int. Ed. Engl. 33, 1579-1581 (1994); (b) R. J. Pieters, I. Huc, J. Rebek, Jr. Tetrahedron 51, 485-498 (1995).

37. D. H. Lee, K. Severin, M. R. Ghadiri. Curr. Opin. Chem. Biol. 1, 491-496 (1997).

38. S. Yao, I. Ghosh, R. Zutshi, J. Chmielewski. Nature 396, 447-450 (1998). 